EPJ Web of Conferences 59, 05016 (2013)

DOI: $10.1051 /$ epjconf/20135905016

(C) Owned by the authors, published by EDP Sciences, 2013

\title{
Investigation of low-coherence laser radiation interaction with different materials
}

\author{
A.A. Fronya ${ }^{a}$, N.G. Borisenko, Yu.A. Merkuliev, M.V. Osipov, V.N. Puzyrev, \\ A.N. Starodub and B.L. Vasin
}

P.N. Lebedev Physical Institute of RAS, 119991 Moscow, Russia

\begin{abstract}
The paper presents the results of experiments on the interaction of low-coherence laser radiation with low density volume-structured materials and solid-state density materials, performed at the facility "KANAL-2" [1]. The data on scattering, absorption, and transport of low-coherence heating laser radiation through plasmas from low-density volume-structured materials and solid density materials have been analyzed. The experiments have demonstrated that laser radiation of low coherence effectively interacts with low density materials, and materials of solid-state density.
\end{abstract}

\section{INTRODUCTION}

The state of the art of laser-matter interaction research is characterized by intensive development of super-high-power laser facilities such as Iskra-6, in Russia; LMJ, in France; NIF, in the USA. The main aim of these facilities is "burning" of the thermonuclear reaction in target with fuel.

The principal feature of such projects is the presence of a target super-compressed by highly homogeneous laser radiation. The achievement of such homogeneity of radiation is a complex scientific and engineering problem, which is being solved, at present, by technically complex and expensive methods (phase plates, adaptive optics, and so on). The uniform distribution of the radiation intensity over the target surface has a significant influence on the parameters of the thermonuclear plasma at the final stage of compression; this is why the homogeneity of the target irradiation is an important factor in the ICF problem.

One way to resolve the above issues is the development of laser systems with a controllable degree of radiation coherence. It is known [1] that the decrease in the degree of spatial coherence of the laser beam leads to suppression of small-scale self-focusing of radiation in the optical laser medium. And temporal coherence affects the gain of the signal propagating in the active medium [2]. Controlling coherence can improve the efficiency of the laser system with simplified requirements for the optical scheme of the laser system. Also for improving the transport and transfer of the laser energy to the plasma, special materials were proposed, as well as using structured targets [3]. An example of such materials are low density volume-structured materials, which are being suggested for homogeneous distribution of laser intensity on target surface [4].

In the present paper the experimental study of backscattered radiation from plasma and radiation transmitted through the plasma are discussed. Plasma was created by low-coherence laser radiation.

\footnotetext{
ae-mail: anastasiya@fronya.com
}

This is an Open Access article distributed under the terms of the Creative Commons Attribution License 2.0, which permits unrestricted use, distribution, and reproduction in any medium, provided the original work is properly cited. 
Table 1. Characteristics of laser radiation.

\begin{tabular}{|l|l|}
\hline Wavelength $\lambda$ & $\sim 1060 \mathrm{~nm}$ \\
\hline Laser pulse duration $\tau$ & $\sim 2,5 \mathrm{~ns}$ \\
\hline Width of laser line $\delta \lambda$ & $42 \AA$ \\
\hline Pulse energy & $10-100 \mathrm{~J}$ \\
\hline Power flux density & $10^{12}-10^{14} \mathrm{~W} / \mathrm{cm}^{2}$ \\
\hline Angle of divergence $\alpha$ & $\sim 10^{-3} \mathrm{rad}$ \\
\hline Number of transverse modes & $\sim 1000$ \\
\hline Pulse radiation contrast & $>10^{6}$ \\
\hline
\end{tabular}

\section{EXPERIMENTAL RESULTS}

The experiments have been performed with the Nd-glass laser facility «KANAL-2». The target was irradiated by one beam of neodymium glass laser radiation (characteristics presented in Table 1). The parameters of laser radiation are not changed in experiments. The spot size of laser radiation focused on the target surface was $\sim 170$ or $350 \mu \mathrm{m}$. The scheme of the laser system allows to control the coherence of radiation: we change the angle of divergence, number of transverse modes, width of laser line, simultaneously, and therefore the degree of coherence is changed. The laser consists of a master oscillator, preamplification system and serial cascade amplifiers. The formation of spatial-temporal characteristics of the laser radiation takes place in the master oscillator, and required for loading of the cascade pre-amplifiers the radiation contrast, and the level of energy at the given pulse duration are provided. In this laser scheme, spatial filters between successive amplification cascades and complex optical devices for laser beam wave front correction are absent. It allows one to significantly simplify the laser scheme and to decrease the total number of optical elements used in the amplifying laser path. For a laser radiation linewidth of $42 \AA$ the coherence time is equal to $8 \cdot 10^{-13}$ seconds.

In the experiments polymer aerogels and solid-state foils were used. Polymer aerogel of triacetate cellulose (TAC) is structured as a 3D network [5]. The density of TAC targets was 50;10;9;4.5; 4; 2.25 and $2 \mathrm{mg} / \mathrm{cm}^{3}$. The target thickness was 100-2000 $\mu \mathrm{m}$. Solid-state targets were metal $(\mathrm{Cu}, \mathrm{Mg}, \mathrm{Gd}, \mathrm{Al})$ foils with thickness from $100 \mu \mathrm{m}$.

Energy of heating laser radiation, backscattering radiation from plasma and radiation transmitted through the plasma were measured. Measurements of backscattering and transmitted energy were performed in limited spatial angle, equal to the spatial angle of the laser focusing system.

For the present experimental scheme (Fig. 1), the energy balance may be written as equation:

$$
E_{l}=E_{a b s}+E_{b s}+E_{f s+t r}+E_{s}
$$

with $E_{l}$ - energy of heating laser radiation, $E_{a b s}$ - energy absorbed by target plasma, $E_{b s}$ - energy of backscattering radiation in aperture of focusing lens, $E_{f s+t r}$ - energy of radiation transmitted through the plasma, registered in limited aperture of optical system, $E_{s}$ - energy of radiation scattered by plasma in another directions and not registered in experimental conditions.

For solid-state target the penetration of laser energy through the plasma and target does not occur at presented experimental conditions, but for TAC targets it was found that through the TAC plasma a considerable part of the laser energy may penetrate (up to 70\% from heating laser energy).

The energy transmitted through the TAC targets decreases with increase in the aerogel thickness $(l)$, with increase in the target density $(\rho)$, with increase in the target linear mass $(\rho l)$ [6]. At increasing the thickness, density and consequently linear mass, the mass matter interacting with laser radiation is rising. As a result the size of the plasma layer and plasma region with electron density close to critical electron density are increased. Near these regions reflection and absorption of the main part of laser energy occurs, and consequently the part of laser energy transmitted through the plasma is decreased. 


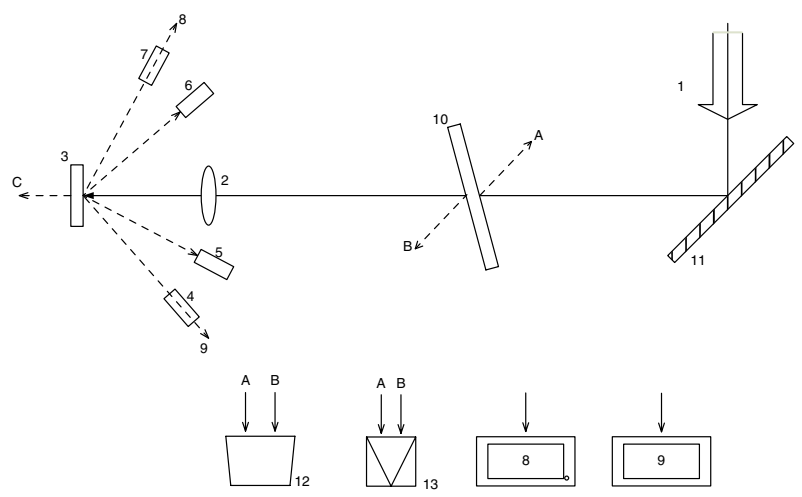

Figure 1. 1 - heating (A), backscattering (B) and transmitted (C) radiation measurements; 2 - focusing system, 3 - target, 4 - step attenuator, 5 - control target system, 6 - four-frequency polarized microscope, 7 - X-ray detector with time resolution, 8 - oscilloscope, 9 - computer, 10 - plane-parallel plate, 11 - mirror, 12 - spectrograph, 13 - calorimeter.
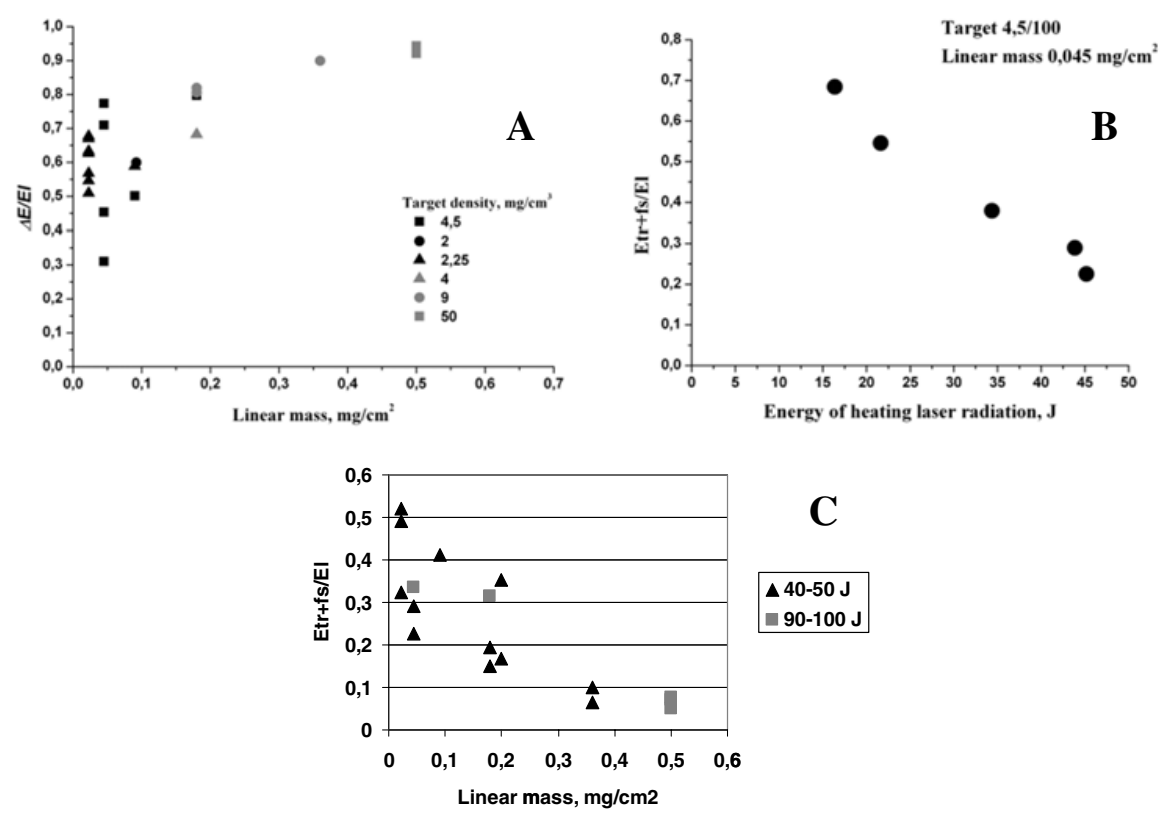

Figure 2. $\mathrm{A}-\Delta E$ dependence on linear mass, $\mathrm{B}$ - dependence of the fraction of heating laser energy transmitted through the plasma on heating laser energy, $\mathrm{C}$ - dependence of the fraction of heating laser energy transmitted through the plasma on TAC target linear mass, for different laser energy intervals.

Also energy $\Delta E$ was estimated:

$$
\Delta E=E_{l}-E_{b s}-E_{f s+t r}=E_{a b s}+E_{s} .
$$

It was revealed that $\Delta E$ is going up with increasing linear mass of TAC target (Fig. 2A). Whereas one component of $\Delta E$ is the energy absorbed by the target, the form of curve characterizing the dependence $\Delta E(\rho l)$ is interpreted as increasing of plasma volume and mass of target matter interacted with laser radiation, in wich absorption process take place. 
Also it was found that for TAC targets with identical density and thickness the part of laser energy transmitted through the plasma is reduced at increasing power flux density of the heating radiation (Fig. 2B). To explain this effect it is needed to adress to electron temperature dependence on laser energy and $\Delta E$ [6]: electron temperature grows with increasing $E_{l}$ and $\Delta E$ and consequently $E_{a b s}$ grows, herewith considerable growth of backscattering energy is not found.

In addition the energy transmitted through the plasma dependences on linear mass were plotted for different laser energy intervals (on Fig. 2.C graphs are presented for 40-50 and 90-100 J intervals). From Fig. 2.C one can see that the part of energy transmitted through the plasma depends on target linear mass, laser energy, as well as other target and laser radiation parameters, and it results in dispersion of experimental data at identical linear mass and laser energy.

It was found that energy of the radiation backscattered into the focusing lens aperture is less than $1 \%$ for all types of targets used in the presented experiments. But for solid-state targets, energy backscattered in the focusing lens aperture is bigger than for TAC targets. In spite of so small a value of backscattering radiation energy, nonlinear processes can not be ignored. This is indicated by the changing of backscattering radiation spectrum (broadening) and the harmonic generation in plasma $\left(2 \omega_{0}, 3 / 2 \omega_{0}\right.$ and $\left.5 / 2 \omega_{0}\right)$.

Detailed study of energy balance dependence on the laser radiation parameters is among our future investigations.

\section{CONCLUSIONS}

The performed laser-matter interaction experiments demonstrated that the low-coherence laser radiation effectively interacts with low density and solid-state materials. At irradiation of low density TAC targets a significant part of laser energy (up to 70\%) penetrates through the plasma created by laser radiation. But the fraction of energy transmitted through the target plasma depends on many factors: parameters of targets and laser radiation. The low coherence laser radiation has a small reflection coefficient. The revealed dependences indicate the nonlinear character of absorption processes and reflection of laser radiation for targets.

The work is partly supported by the Russian Foundation for Basic Researches, grant \# 10-02-00113 and by Federal Target Program "Research and scientific-pedagogical cadres of Innovative Russia" (grant 2009-1.1-122-052-025).

\section{References}

[1] S.I. Fedotov, L.P. Feoktistov, M.V. Osipov, and A.N. Starodub, Journal of Russian Laser Research 25, 72 (2004)

[2] N. Blanchot, C. Rouyer, C. Sauteret and A. Migus, Optics Letters 20, 395 (1995)

[3] N.G. Borisenko, Yu.A. Merkul'ev, A.I. Gromov, Journal of the Moscow Physical Society 4, 247 (1994)

[4] I.N. Voronich, S.G. Garanin, V.N. Derkach, A.I. Zaretskii, A.G. Kravchenko et al., Quantum Electronic. 31, 370 (2001)

[5] N.G. Borisenko, I.V. Akimova, A.I. Gromov, A.M. Khalenkov, V.N. Kondrashov, J. Limpouch, E. Krousky, J. Kuba, K. Masek, Yu.A. Merkuliev, W. Nazarov, V.G. Pimenov, Fusion Science and Technology 49, 676 (2006)

[6] A.N. Starodub, N.G. Borisenko, A.A. Fronya, Yu.A. Merkuliev, M.V. Osipov, V.N. Puzyrev, A.T. Sahakyan, B.L. Vasin, O.F. Yakushev, Laser and Particle Beams 28, 371 (2010) 